\title{
Association of sexual assault history with traumatic childbirth and subsequent PTSD
}

\author{
Zohar Berman $^{1,2} \cdot$ Freya Thiel $^{1,3} \cdot$ Anjali J. Kaimal ${ }^{4,5} \cdot$ Sharon Dekel ${ }^{1,2}$ \\ Received: 15 September 2020 / Accepted: 3 April 2021 / Published online: 13 April 2021 \\ (c) The Author(s), under exclusive licence to Springer-Verlag GmbH Austria, part of Springer Nature 2021
}

\begin{abstract}
Although childbirth-related posttraumatic stress (CB-PTSD) has received recognition, how sexual assault (SA) history influences obstetrical and traumatic stress outcomes remains unclear. Six hundred eighty-three women provided information about their childbirth and mental health. Obstetric complications and unplanned cesareans were more prevalent among women with SA history. They also had higher rates of probable CB-PTSD and were two times more likely to have premature deliveries than women without SA history. Screening women for history of sexual trauma is warranted to optimize birth outcomes.
\end{abstract}

Keywords Postpartum posttraumatic stress disorder $\cdot$ Trauma $\cdot$ Childbirth $\cdot$ Preterm delivery $\cdot$ Maternal morbidity . Psychiatric

\section{Introduction}

Childbirth is a complex event. Up to one third of women report a traumatic experience and a significant minority can go on and develop a traumatic stress disorder. This condition, coined childbirth-related posttraumatic stress disorder (CB-PTSD), may affect maternal and child health(Dekel et al. 2017). Sexual assault (SA) experienced by up to 1 out of 5 American women involves sexual contact or behavior without the consent of the recipient. SA can increase risk for subsequent traumatic stress-related disorders as evident in non-postpartum samples(Dworkin et al. (2017)). However, the relationship between SA history and traumatic childbirth remains unclear.

Sharon Dekel

sdekel@mgh.harvard.edu

1 Department of Psychiatry, Massachusetts General Hospital, 120 2nd Ave, Boston, MA, USA

2 Department of Psychiatry, Harvard Medical School, Boston, MA, USA

3 Present Address: Department of Medicine, Faculty of Human Sciences, MSH Medical School Hamburg, Hamburg, Germany

4 Department of Obstetrics and Gynecology, Massachusetts General Hospital, Boston, MA, USA

5 Department of Obstetrics, Gynecology, and Reproductive Biology, Harvard Medical School, Boston, MA, USA
Qualitative studies suggest that childbirth can trigger memories of sexual trauma and may provoke fear and dissociative responses in women with SA history(Halvorsen et al. 2013). Quantitative studies largely focused on depressive responses and there is work in support of increased prevalence of postpartum depression among women with SA history(Wosu et al. 2017), but much less is known regarding traumatic stress reactions to childbirth. Studies examining the subjective experience of childbirth do show that women with SA are more likely to experience fear and dissociation in childbirth(Zambaldi et al. (2011)) and SA history has been identified as among the risk factors implicated in CB-PTSD(Chan et al. (2020)) although it remains unclear whether factors such as demographics may account for this relationship. To the best of our knowledge, no study has directly compared acute stress and CB-PTSD in women with and without SA history while considering potential differences in background factors.

Quantitative studies further support an association between SA and adverse obstetrical outcomes such as preterm delivery(Wosu et al. 2015), which can pose risk to infant health. Other stressors in childbirth, such as prolonged duration of labor, increased pain in birth, obstetric complications, and medical interventions pertaining to cesarean section, have been consistently linked with SA history in some studies but not others(Schei et al. 2014; Hulst et al. 2006).

To better understand this relationship, we assessed the impact of SA history on obstetrical and birth-related 
traumatic stress outcomes in a large sample of women following childbirth.

\section{Methods}

\section{Procedure and participants}

This is a secondary analysis of a project on childbirthrelated psychological outcomes(Dekel et al. 2019). Women who gave birth in the past 6 months to a live baby and were at least 18 years old were recruited via postpartum websites (e.g., Postpartum Progress) between November 2016 and April 2017. Consent was implied by completing the anonymous online survey. Partners (Mass Genral Brigham) Healthcare Human Research Committee's granted the study exemption.

\section{Measures}

Sexual assault history was assessed using the Life Events Checklist for DSM-5 (LEC-5) pertaining to items of SA and determined based on reporting that the experience directly "happened to me."

Childbirth-related acute distress during/immediately after childbirth was assessed using the well-validated Peritraumatic Distress Inventory (PDI) and Peritraumatic Dissociative Experiences Questionnaire (PDEQ). The PDI measures acute stress reactions to a specific trauma (here, recent childbirth) using 13 items (e.g., "feeling helpless") $(\alpha=0.89)$. The cutoff of $\geq 23$ indicates clinically significant stress. The PDEQ assesses dissociative responses (here, to childbirth) with 10 items (e.g., event "seemed unreal to me") $(\alpha=0.91)$.

Childbirth-related PTSD was assessed with the PTSD Checklist for DSM-5 (PCL-5), which is the most commonly used self-report to measure 20 PTSD symptoms in regard to a specific event ("recent childbirth") ( $\alpha=0.95)$. To conform with DSM-5 PTSD criteria, we classified individuals as endorsing probable CB-PTSD by having at least 1 intrusion, 1 avoidance, 2 alterations in cognitions and mood, and 2 reactivity and hyperarousal, with at least moderate severity.

Background information collected via single items included demographics (maternal age, primiparity, education, income, marital status, ethnicity), prior PTSD (report of PTSD before childbirth), and pregnancy history (i.e., miscarriages/premature deliveries/ stillbirths).

Information concerning recent childbirth was collected by self-report and pertained to labor and delivery pain, medication for pain and labor induction, duration of labor (i.e., time between contractions and birth), obstetric complications in labor/delivery (single item, yes vs no), prematurity $(<37$ gestational week), and delivery mode.

\section{Data analyses}

Missing data was estimated using Little's Missing Completely At Random (MCAR) test and handled using Multiple Imputation. Independent $t$-tests for continuous variables and chi-square, Fisher's exact tests, and odds ratio (for effect sizes) for categorical variables were used to examine differences between women with and without SA history on main measures. One-tailed tests accorded with previous literature suggests of directional hypotheses (distress/dissociation, labor duration, labor/delivery pain, unscheduled cesarean, obstetrical complications, prematurity). Analyses of covariance (ANCOVA) were employed to examine group differences in traumatic stress controlling for background factors that differed between study groups and contribute to PTSD (i.e., age, income, education, prior PTSD)(Chan et al. 2020).

\section{Results}

The sample in the large project comprised of 685 women who were on average 3 months postpartum (out of 795 eligible and 110 excluded due to missing data)(Dekel et al. 2019). Additionally, two participants were excluded from this study due to missing SA status information. Twenty percent of the sample (137/683) reported SA history. These women were younger and less educated, had lower income, and reported more prior PTSD than women without SA history (Table 1). Women with prior PTSD had more preterm deliveries $(p=0.032)$. Of those who endorsed probable CB-PTSD, most $(81 \%, 110 / 136)$ denied PTSD before childbirth.

Pregnancy history. The SA group had higher rates of previous miscarriage and preterm delivery than controls. No group differences in stillbirth were observed (Table 1).

Recent childbirth. The SA group had higher prevalence of obstetrical complications and unplanned cesareans and they were nearly two times as likely to report preterm delivery than the no SA group controlling for prior PTSD ( $p=0.033)$. No differences in obstetrical outcomes by demographics (maternal age, income, education: complications vs. non, $p=0.996, p=0.31, p=0.06$; cesarean vs. other $=p=0.06, p=0.37, p=0.47$; preterm vs. no, $p=0.99, p=0.87, p=0.75)$. The SA group had higher prevalence of clinically significant acute stress in childbirth and CB-PTSD (Table 1). Differences remained excluding participants with prior PTSD $(O R=2.54, C I=1.58-4.08)$. ANCOVAs accounting for background factors revealed 
Table 1 Demographics, pregnancy history, recent childbirth, and traumatic stress by sexual assault status

\begin{tabular}{llllll}
\hline Variable & $\begin{array}{l}\text { SA } \\
(n=137)\end{array}$ & $\begin{array}{l}\text { No SA } \\
(n=546)\end{array}$ & $p$ & OR & $95 \%$ CI \\
& M (SD)/\% & M (SD)/\% & & & \\
& & & & & \\
Background & $30.07(4.7)$ & $31.6(4.6)$ & .000 & & \\
$\quad$ Age (years) & 88.3 & 89.9 & .638 & 0.85 & $0.47-1.53$ \\
Ethnicity (non-Hispanic White) & 53.3 & 75.8 & .000 & 0.36 & $0.25-0.54$ \\
Education (Bachelor's degree and higher) & 60 & 78.4 & .000 & 2.43 & $1.63-3.62$ \\
Income (<50 K) & 89.1 & 94.1 & .057 & 0.51 & $0.27-0.97$ \\
$\quad$ Marital status (married/cohabiting) & 53.3 & 56.6 & .502 & 0.88 & $0.60-1.27$ \\
Primiparity (primiparas) & 21.2 & 4.6 & .000 & 5.6 & $3.15-9.93$ \\
Prior PTSD (endorsed) & & & & & \\
Pregnancy history & 31.4 & 22 & .025 & 1.62 & $1.07-2.46$ \\
Miscarriage & 4.9 & 10.2 & .027 & 2.19 & $1.11-4.3$ \\
Preterm & 2.2 & 1.5 & .468 & 1.51 & $0.39-5.75$ \\
Stillbirth & & & & & \\
Recent childbirth & $3.37(1.40)$ & $3.42(1.34)$ & .347 & - & - \\
Pain during labor (M/SD) & $2.65(1.44)$ & $2.71(1.43)$ & .333 & - & - \\
Pain during delivery (M/SD) & 78.1 & 74.9 & .505 & 1.20 & $0.76-1.87$ \\
Medication for pain & 38.7 & 43.4 & .335 & 0.82 & $0.56-1.21$ \\
Medication for induction of labor & $18.93(60.52)$ & $15.13(22.39)$ & .236 & - & - \\
Duration of labor (hours) (M/SD) & 54.7 & 45.4 & .032 & 1.45 & $1.00-2.12$ \\
Obstetric complications in labor or delivery & & 18.7 & .028 & 1.67 & $1.03-2.71$ \\
Unplanned cesarean (vs. vaginal) & 27.8 & 8.6 & .017 & 1.92 & $1.11-3.34$ \\
Preterm devliery & 15.3 & & & \\
Mental health & 35.8 & 27.2 & .032 & 1.49 & $1.00-2.22$ \\
Acute stress in birth & 34.3 & 16.3 & .000 & 2.68 & $1.76-4.08$ \\
Childbirth-related PTSD (CB-PTSD) & & & & & \\
\hline
\end{tabular}

$S A$ sexual assault history. Prior PTSD refers to endorsement of PTSD before recent childbirth. $M$ mean, $S D$ standard deviation, $O R$ odds ratio, $C I$ confidence interval. $N=683$ group differences in acute stress in childbirth (SA, PDI: $M=18.97, S D=12.62$; no SA, PDI: $M=15.29, S D=11.96$ ), dissociation (SA, PDEQ: $M=13.33, S D=10.37$; no SA, PDEQ: $M=10.07, S D=9.27$ ), and CB-PTSD (SA, PCL5: $M=29.80, S D=21.03$; no SA, PCL-5: $M=19.59$, $S D=16.78)$ (Table 2).

\section{Discussion}

We found that women with SA history reported more obstetrical complications and more frequently had unplanned cesareans and they were two times as likely to have premature deliveries than women without a history of SA. These differences were revealed in an educated potentially high resource sample. Controlling for demographic factors such as age, education, income, and prior PTSD, we found that having SA history was associated with clinically significant traumatic stress responses to childbirth. Women with SA had more acute stress in childbirth and probable CB-PTSD, which were assessed by measures that are used to determine traumatic stress following other traumas (e.g., combat).

The obstetrical stressors prevalent among women with SA history in this study, mainly unplanned cesarean and premature birth, can have adverse health implications. Premature delivery is associated with severe health complications in the newborn and medical costs. Unplanned cesarean may increase risk for maternal mental health problems(Dekel et al. 2019; Houston et al. 2015).

Our findings of heightened acute stress and dissociation in birth in women with SA history are similar to the findings of previous studies(Hulst et al. 2006). It has been suggested that childbirth can trigger the experience of retraumatization for SA survivors, and manifest in a sense of defeat, loss of control, and objectification. This experience may influence obstetrical outcomes and is partly associated with birth-related procedures. Our study furthers shows that SA history is associated with the onset of PTSD following childbirth, suggesting increased risk for maternal psychiatric morbidity among traumatized women. The findings accord 
Table 2 Differences in CB-PTSD, traumatic acute stress response to childbirth, and dissociation by sexual assault status controlling for background factors

\begin{tabular}{|c|c|c|c|c|c|c|}
\hline Variable & Source & SS & $d f$ & MS & $F$ & $p$ \\
\hline \multirow{8}{*}{$\begin{array}{l}\text { Childbirth-related } \\
\text { PTSD (CB-PTSD) }\end{array}$} & Corrected model & $27,271.83$ & 5 & 5454.37 & 18.67 & .000 \\
\hline & Intercept & $14,640.56$ & 1 & $14,640.56$ & 18.67 & .000 \\
\hline & Age & .309 & 1 & .31 & .001 & .487 \\
\hline & Income & 3102.45 & 1 & 3102.45 & 10.62 & .000 \\
\hline & Education & 709.55 & 1 & 709.55 & 2.43 & .060 \\
\hline & Prior PTSD & 7020.78 & 1 & 7020.78 & 24.04 & .000 \\
\hline & Sexual assault & 4025.13 & 1 & 4025.13 & 13.78 & .000 \\
\hline & Error & $197,739.42$ & 677 & 292.08 & & \\
\hline \multirow[t]{8}{*}{ Acute stress in birth } & Corrected model & 3548.39 & 5 & 709.68 & 4.93 & .000 \\
\hline & Intercept & 5563.32 & 1 & 5563.32 & 38.62 & .000 \\
\hline & Age & 82.30 & 1 & 82.30 & .571 & .225 \\
\hline & Income & 34.99 & 1 & 34.99 & .243 & .311 \\
\hline & Education & 2.29 & 1 & 2.29 & .016 & .450 \\
\hline & Prior PTSD & 1733.26 & 1 & 1733.26 & 12.03 & .001 \\
\hline & Sexual assault & 550.70 & 1 & 550.70 & 3.82 & .026 \\
\hline & Error & $97,557.15$ & 677 & 144.10 & & \\
\hline \multirow[t]{8}{*}{ Dissociation in birth } & Corrected model & 2749.34 & 5 & 549.87 & 6.22 & .000 \\
\hline & Intercept & 3748.84 & 1 & 3748.84 & 42.40 & .000 \\
\hline & Age & 94.09 & 1 & 94.09 & 1.06 & .152 \\
\hline & Income & 45.45 & 1 & 45.45 & .514 & .237 \\
\hline & Education & 137.76 & 1 & 137.76 & 1.56 & .106 \\
\hline & prior PTSD & 791.83 & 1 & 791.83 & 8.96 & .002 \\
\hline & Sexual assault & 418.89 & 1 & 418.89 & 4.74 & .015 \\
\hline & Error & $59,852.86$ & 677 & 88.41 & & \\
\hline
\end{tabular}

$S S$ sum of squares, $M S$ means. One-tailed $p$ values with the notion that exposure to trauma increases vulnerability for PTSD in future traumas.

There are several limitations worth noting. We assessed current PTSD with the commonly used self-report measure but not with clinical interviews. Information about childbirth was obtained retrospectively and can involve memory bias and we did not ask about specific obstetrical factors. We cannot make clear-cut conclusions whether CB-PTSD signifies new PTSD or amplification of prior PTSD, although the vast majority of participants with CB-PTSD denied endorsing PTSD history. The association between SA and obstetrical factors may be accounted for by other (demographic) factors and we cannot assume cause and effect. SA and PTSD history concerned lifetime rather than specific time periods (e.g., childhood). The web recruitment possibly targeted more affected women that connect SA with negative outcomes, and the sample represents educated White women, which may raise concerns regarding generalizability.

In conclusion, 4 million women give birth in the US each year, of which expected 800,000 may have experienced sexual trauma. These findings suggest that screening pregnant women for SA histories may help to identify a population at risk for adverse outcomes. Further work is needed to understand what interventions may be offered to optimize both childbirth experiences and longterm outcomes. Broadening the assessment of peripartum mental health, especially during the recent COVID-19 pandemic(Mayopoulos et al. 2021), to include disorders of traumatic stress in routine care may help to identify opportunities to better optimize maternal and neonatal mental and physical health.

Acknowledgements The authors would like to thank Ms. Shannon Hennig for her generous support of this research project. We also would like to thank Ms. Gabriella Dishy for assisting in developing the Internet survey, and Ms. Sabrina Chan for assisting in manuscript preparation.

Author contribution $\mathrm{ZB}$ conducted the main data analysis and wrote the initial manuscript text. FT contributed to the writing of the "Methods" and "Results" sections and the editing the manuscript. AJK helped with the preparation of the study manuscript and provided final editing. $\mathrm{SD}$ is the principal investigator of the larger project. She designed the current study, supervised the manuscript writing and preparation, and provided significant editing of the final version. All authors read and approved the manuscript.

Funding Dr. Dekel was supported by awards from the National Institute of Child Health and Human Development (R21HD100817) and the 
Mass General Executive Committee on Research (ECOR) (ISF award). The sponsor had no involvement or role in the study.

\section{Declarations}

Ethics approval This study was provided exempt by the Partners (Massachusetts General Hospital) Human Research Committee It involved an annoymous study survey.

Conflict of interest The authors declare no competing interests.

\section{References}

Dekel S, Stuebe C, Dishy G (2017) Childbirth induced posttraumatic stress syndrome: a systematic review of prevalence and risk factors. Front Psychol 8:560

Dworkin ER, Menon SV, Bystrynski J, Allen NE (2017) Sexual assault victimization and psychopathology: a review and meta-analysis. Clin Psychol Rev 56:65-81

Halvorsen L, Nerum H, Oian P, Sørlie T (2013) Giving birth with rape in one's past: a qualitative study. Birth 40:182-191

Wosu A, Gelaye B, Willians A (2017) History of childhood sexual abuse and risk of prenatal and postpartum depression or depressive symptoms: an epidemiologic review. Arch Womens Ment Health 18:659-671

Zambaldi CF, Cantilino A, Farias JA, Moraes GP, Sougey EB (2011) Dissociative experience during childbirth. J Psychosom Obstet Gynaecol 32:204-209
Chan SJ, Ein-Dor T, Mayopoulos PA, Mesa MM, Suda RM, McCarthy BF, Kaimal A, Dekel S (2020) Risk factors for developing posttraumatic stress disorder following childbirth. Psychiatry Res 290:113090

Wosu A, Gelaye B, Williams M (2015) Maternal history of childhood sexual abuse and preterm birth: an epidemiologic review. BMC Pregnancy Childbirth 15:174

Schei B, Lukasse M, Ryding EL, Campbell J, Karro H, Kristjansdottir $\mathrm{H}$ et al (2014) A history of abuse and operative delivery-results from a European multi-country cohort study. PLoS ONE 9:e87579

van der Hulst LA, Bonsel GJ, Eskes M, Birnie E, van Teijlingen E, Bleker OP (2006) Bad experience, good birthing: Dutch low-risk pregnant women with a history of sexual abuse. J Psychosom Obstet Gynecol 27:59-66

Dekel S, Ein-Dor T, Berman Z, Barsoumian IS, Agarwal S, Pitman RK (2019) Delivery mode is associated with maternal mental health following childbirth. Arch Women's Ment Health 22:817-824

Houston KA, Kaimal AJ, Nakagawa S, Gregorich SE, Yee LM, Kuppermann M (2015) Mode of delivery and postpartum depression: the role of patient preferences. Am J Obstet Gynecol 212(229):e17. https://doi.org/10.1016/j.ajog.2014.09.002

Mayopoulos PA, Ein-Dor T, Dishy GA, Nandru R, Chan SJ, Hanley LE, Kaimal A, Dekel S (2021) COVID-19 is associated with traumatic childbirth and subsequent mother-infant bonding problems. J Affect Disord 282:122-125. https://doi.org/10.1016/j.jad.2020. 12.101

Publisher's note Springer Nature remains neutral with regard to jurisdictional claims in published maps and institutional affiliations. 